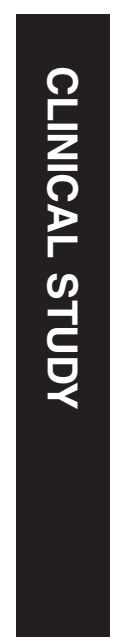

\title{
Three percent diquafosol ophthalmic solution as an additional therapy to existing artificial tears with steroids for dry-eye patients with Sjögren's syndrome
}

Department of

Ophthalmology, Kyoto Prefectural University of Medicine, Kyoto, Japan

Correspondence: N Yokoi, Department of Ophthalmology, Kyoto Prefectural University of Medicine, 465 Kajii-cho, Hirokoji-agaru, Kawaramachidori, Kamigyo-ku, Kyoto 602-0841, Japan Tel: +8175 251 5578; Fax: +81752515663. E-mail: nyokoi@ koto.kpu-m.ac.jp

Received: 16 December 2014

Accepted in revised form: 15 May 2015 Published online: 10 July 2015

This study was previously presented in part as a poster at the Tear Film and Ocular Surface Society Meeting in Taormina, Italy (September 19-22, 2013).

\begin{abstract}
Purpose To investigate the long-term results of $3 \%$ diquafosol ophthalmic solution as an alternative therapy to existing ophthalmic solutions, including topical immunosuppression, for the treatment of dry eye in patients with Sjögren's syndrome.

Methods This study involved 14 female dryeye patients (mean age: 62.4 years) with Sjögren's syndrome who insufficiently responded to their current therapy. In all patients, 3\% diquafosol ophthalmic solution was administered six times daily for 12 months in substitution for artificial tears and sodium hyaluronate ophthalmic solution. Their use of corticosteroid eye drops remained unchanged from that prior to the treatment with diquafosol sodium. The subjective symptoms assessed, and ocular signs including tear meniscus radius and the tear film breakup time, and ocular-surface epithelial damage score were examined at 1 , 2, 3, 4, 5, 6, 9, and 12 months after initiating treatment.

Results Among the subjective symptoms, significant improvement was obtained in dryness at 2 months post treatment, in eye fatigue at 1, 2, 3, 4, and 12 months post treatment, and in pain at 1, 2, 6, and 12 months post treatment. Difficulty in opening the eye, foreign body sensation, and redness were also significantly ameliorated at various time-points. The tear meniscus radius and the tear film breakup time were significantly improved throughout the observation period, and the corneal epithelial staining scores were significantly decreased at 3 months post treatment.
\end{abstract}

N Yokoi, Y Sonomura, H Kato, A Komuro and $S$ Kinoshita

Conclusions In dry-eye patients with Sjögren's syndrome, treatment with $3 \%$ diquafosol ophthalmic solution improved both symptoms and signs, and that effectiveness was maintained for 12 months. Eye (2015) 29, 1204-1212; doi:10.1038/eye.2015.125; published online 10 July 2015

Introduction

According to the International Dry Eye Workshop (DEWS) classification, dry eye is classified into one of the following two types: (1) aqueous tear-deficient dry eye and (2) evaporative dry eye, and Sjögren's syndrome (SS) is reportedly associated with the former. ${ }^{1}$ SS is an organ-specific autoimmune disorder that mainly targets the lacrimal and salivary glands. This disease predominantly occurs in females, and is characterized by mononuclear cells infiltrating into and destroying lacrimal and salivary glands, resulting in decreased secretory function, which leads to keratoconjunctivitis sicca and xerostromia. ${ }^{2}$ It is reported that relatively severe ocular-surface epithelial damage is the main ocular manifestation of SS, which may be associated with decreased tear fluid secretion and inflammation on the ocular surface. $^{3-7}$ Moreover, several studies have reported a decrease of ocular-surface goblet cell density and mucin 5AC (MUC5AC) level in cases of dry eye with SS. 8,9

A 3\% diquafosol ophthalmic solution, which was launched for the treatment of dry eye in Japan, reportedly promotes water secretion, ${ }^{10-13}$ secretory mucin secretion, ${ }^{14,15}$ and membraneassociated mucin expression ${ }^{16}$ on the ocular 
surface, thus resulting in the improvement of dry-eyerelated signs and symptoms. ${ }^{17-22}$

Diquafosol sodium exhibits a $\mathrm{P}_{2} \mathrm{Y}_{2}$ agonist activity that accelerates fluid transport from the serosal to mucosal (tear) side via chloride channel activation after intracellular calcium ion concentration elevation in the conjunctival epithelium. ${ }^{23,24}$ This action has been demonstrated in normal rat, ${ }^{10}$ rabbit, $^{11}$ and cat ${ }^{12}$ experimental models in which the Schirmer test value and tear meniscus area increased after topical application of the diquafosol ophthalmic solution. As it has also been shown to facilitate tear-secretion increase in a rat dry-eye model in which the exorbital lacrimal glands were extirpated, ${ }^{10}$ this mode of action may be independent of the lacrimal glands. Thus, it was theorized that diquafosol ophthalmic solution facilitates tear fluid secretion and ameliorates the signs and symptoms of dry eye with SS in comparison with the conventional treatments such as the application of sodium hyaluronate and artificial tears. ${ }^{25,26}$

The purpose of this present study was to evaluate the long-term results of 3\% diquafosol ophthalmic solution for the treatment of dry eye with SS when substituted for the standard treatments of artificial tears and sodium hyaluronate.

\section{Materials and methods}

\section{Subjects}

Approval for this study was obtained from the Institutional Review Board of Kyoto Prefectural University of Medicine, Kyoto, Japan prior to initiation. The research was conducted in accordance with the tenets set forth in the Declaration of Helsinki, and prior written informed consent was obtained from all subjects after receiving a detailed explanation of the nature of the study and possible consequences associated with participation in the study.

Enrolled in this study were 14 female SS patients (mean age: $62.4 \pm 8.5$ years; mean $\pm S D$ ) in which the disease was diagnosed based on Fox's criteria. ${ }^{27}$ The systemic conditions of the enrolled patients were well controlled by their respective rheumatologists during the study period. In all patients, the eye with the more severe ocular-surface epithelial damage was enrolled, yet if both eyes exhibited the same score, the right eye was selected. Diagnosis of dry eye was established according to the Japanese dry eye diagnostic criteria as follows: (1) presence of dry-eye symptoms, (2) presence of qualitative or quantitative disturbance of the tear film (a Schirmer 1 test value of $\leq 5 \mathrm{~mm} / 5 \mathrm{~min}$, or a tear-film breakup time (BUT) of $\leq 5 \mathrm{~s}$ ), and (3) the presence of corneoconjunctival epithelial damage (a total staining score of $\geq 3$ points). ${ }^{28}$ The presence of all three criteria was necessary for enrollment in the study.

\section{Treatment protocol}

All subjects had been receiving instillation of an artificial tear solution (Soft Santear; Santen Pharmaceutical Co., Ltd., Osaka, Japan) 7 times daily (9 subjects) or 10 times daily (5 subjects), and 4 subjects additionally received $0.1 \%$ sodium hyaluronate (Hyalein; Santen Pharmaceutical) 6 times daily for more than 3 months prior to enrollment in this study. They were treated with $3 \%$ diquafosol ophthalmic solution (Diquas ophthalmic solution 3\%; Santen Pharmaceutical) 6 times daily for 12 months in substitution for artificial tears and $0.1 \%$ sodium hyaluronate. All subjects were permitted to receive ophthalmic corticosteroids $(0.1 \%$ fluorometholone or $0.1 \%$ betamethasone sodium phosphate) once or twice per day only when the symptoms worsened. According to the interview of the patients, they mainly used it in season of dryness. The examinations listed below were performed at 1, 2, 3, 4, 5, 6, 9, and 12 months after the start of treatment, and each parameter was compared to that prior to the treatment with diquafosol sodium.

\section{Evaluation of subjective symptoms}

In this study, before the examination of the ocular surface, a questionnaire comprising 12 dry-eye-related ocular subjective symptoms was used to evaluate the severity of subjective symptoms of each subject, as described in the previous report, ${ }^{29}$ including dryness, difficulty in opening the eye, foreign body sensation, pain, redness, tearing, discharge, itchiness, blurred vision, sensitivity to light, heavy eyelids, and eye fatigue. These symptoms were assessed using a 100-mm visual analog scale (VAS).

\section{Ocular surface examinations}

As noninvasive tear tests, measurement of the radius of the tear meniscus curvature using a videomeniscometer ${ }^{30-33}$ and measurement of the noninvasive BUT (NIBUT) $)^{33,34}$ of the precorneal tear film using a tearfilm lipid layer interferometry system (DR-1; Kowa, Tokyo, Japan) were performed. As invasive ocular surface examinations, the measurement of fluorescein BUT (FBUT) and scoring of ocular surface epithelial damage were performed. All examinations were performed in the order described below.

\section{Tear meniscus radius and noninvasive BUT measurements}

First, the tear meniscus radius of the central lower tear meniscus was measured using a video-meniscometer ${ }^{30,32,33}$ as an index of the total aqueous tear volume over the ocular surface. In this examination, an illuminated target 
comprising a series of horizontal stripes was projected onto the central lower tear meniscus and the specular reflex image of the target was recorded using a digital video recorder. Image analysis software was then used to calculate the radius of the tear meniscus curvature (mm) by application of the concave mirror formula. In order to avoid any effect resulting from instillation of $3 \%$ diquafosol ophthalmic solution, which is known to possibly increase the tear volume up to as late as 60-min post instillation, ${ }^{13}$ the subjects were prohibited from using the solution for at least $1 \mathrm{~h}$ prior to the examination.

Next, NIBUT was measured by use of the DR-1, in which the precorneal tear film is observed within a 6.8-mm (vertical) $\times$ 8.8- $\mathrm{mm}$ (horizontal) rectangular area at the center of the cornea. After several natural blinks, NIBUT was measured as the time (in s) until the first appearance of a full thickness breakup of precorneal tear film within the observed area when the eye is kept open until $10 \mathrm{~s}$. When no noninvasive breakup was observed, $10 \mathrm{~s}$ was used as the NIBUT. The NIBUT was measured only once, since as compared with the FBUT, it generally takes more time for the breakup to occur, thus possibly resulting in triggering reflex tearing, which can lead to incorrect readings in the following FBUT examination.

\section{FBUT measurement and ocular surface epithelial damage scoring}

Next, for the measurement of FBUT and scoring of the ocular surface epithelial damage, a slit-lamp microscope with a cobalt blue filter and blue-free filter ${ }^{35}$ was used. First, two drops of saline solution were put on a fluorescein test strip (Showa Yakuhin Kako Co., Tokyo, Japan), and the strip was then shaken vigorously.
Strict attention was paid to not increase the subject's tear volume by softly touching the strip to the central lower lid margin to stain the ocular surface with fluorescein. After several natural blinks, the FBUT was then measured as the time (in s) until the first appearance of a dark spot in the precorneal tear film when the eye is kept open. FBUT was measured three times and then averaged. Next, scoring of the ocular surface epithelial staining was performed by dividing the cornea into five portions in accordance with NEI scoring system ${ }^{36}$ and the staining was scored from 0 to 3 at each portion to calculate the total score. To assess the conjunctival epithelial fluorescein staining, the nasal and temporal bulbar conjunctiva was evaluated in accordance with the van Bijsterveld scoring system ${ }^{37}$ and the staining was scored from 0 to 3 at each portion to calculate the total score. The overall amounts of corneal and conjunctival epithelial damage were then scored on scales of 0-15 and 0-6 points, respectively.

\section{Statistical analysis}

Paired $t$-tests were used for statistical comparison of the data including subjective symptoms, tear meniscus radius, NIBUT, and FBUT. Wilcoxon's signed rank test was used for the ocular surface epithelial staining scores.

\section{Results}

\section{Patient characteristics}

The baseline demographic information of the patients is summarized in Table 1. Of the 14 patients, 9 patients had primary SS, 5 patients had secondary SS, 8 cases showed a Schirmer test value of $\leq 5 \mathrm{~mm} / 5 \mathrm{~min}$, and 9 cases showed

Table 1 Baseline demographic information of patients in this study

\begin{tabular}{|c|c|c|c|c|c|c|c|c|c|c|c|}
\hline & Age & $\operatorname{Sex}$ & Eye & 1 or $2 S S$ & Complication & FBUT (s) & $\begin{array}{l}\text { Schirmer's } \\
\text { test }(\mathrm{mm})\end{array}$ & OSED (0-9) & $R$ value (mm) & NIBUT (s) & Eyedrops \\
\hline 1 & 62 & $\mathrm{~F}$ & $\mathrm{R}$ & Secondary & RA & 2.0 & 1 & 7 & 0.109 & 2.3 & AT, FL, LVFX \\
\hline 2 & 62 & $\mathrm{~F}$ & $\mathrm{R}$ & Primary & & 0.0 & 2 & 8 & 0.164 & 0.0 & AT, BP, LVFX \\
\hline 3 & 47 & $\mathrm{~F}$ & $\mathrm{R}$ & Primary & & 0.0 & 4 & 9 & 0.218 & 0.0 & AT, HA, FL, LVFX \\
\hline 4 & 69 & $\mathrm{~F}$ & $\mathrm{~L}$ & Primary & & 3.0 & 0 & 7 & 0.055 & 1.7 & AT, FL, LVFX \\
\hline 5 & 68 & $\mathrm{~F}$ & $\mathrm{~L}$ & Primary & & 2.0 & 9 & 8 & 0.136 & 0.0 & AT, HA, FL, LVFX \\
\hline 6 & 55 & $\mathrm{~F}$ & $\mathrm{R}$ & Primary & & 1.7 & 9 & 7 & 0.136 & 3.8 & AT, HA, FL, OFLX \\
\hline 7 & 70 & $\mathrm{~F}$ & $\mathrm{~L}$ & Primary & & 3.0 & 8 & 4 & 0.245 & 3.5 & AT, FL, OFLX \\
\hline 8 & 73 & $\mathrm{~F}$ & $\mathrm{~L}$ & Secondary & RA & 1.3 & 1 & 7 & 0.164 & 0.0 & $\mathrm{AT}, \mathrm{BP}$ \\
\hline 9 & 63 & $\mathrm{~F}$ & $\mathrm{R}$ & Secondary & SSc & 1.3 & 4 & 6 & 0.164 & 0.0 & AT, HA, FL, OFLX \\
\hline 10 & 68 & $\mathrm{~F}$ & $\mathrm{~L}$ & Primary & & 0.0 & 23 & 3 & 0.277 & 0.0 & AT, FL, LVFX, SCG \\
\hline 11 & 54 & $\mathrm{~F}$ & $\mathrm{R}$ & Secondary & $\mathrm{AIH}$ & 2.0 & 4 & 8 & 0.245 & 0.0 & AT, FL, LVFX \\
\hline 12 & 69 & $\mathrm{~F}$ & $\mathrm{R}$ & Primary & & 0.0 & 10 & 7 & 0.191 & 0.0 & AT, FL, OFLX \\
\hline 13 & 47 & $\mathrm{~F}$ & $\mathrm{~L}$ & Secondary & RA & 3.0 & 22 & 8 & 0.204 & 3.7 & AT, FL, OFLX \\
\hline 14 & 67 & $\mathrm{~F}$ & $\mathrm{~L}$ & Primary & & 0.0 & 0 & 9 & 0.095 & 0.0 & AT, FL, LVFX \\
\hline
\end{tabular}

Abbreviations: AIH, autoimmune hepatitis; AT, artificial tear; BP, betamethasone phosphate; F, female; FL, fluorometholone; FBUT, fluorescein breakup time; HA, sodium hyaluronate; LFVX, levofloxacin; NIBUT, noninvasive breakup time; OSDE, ocular surface epithelial damage; R, radius of curvature of tear meniscus; RA, rheumatoid arthritis; SS, Sjögren's syndrome; SSc, systemic screlosis; SCG, sodium cromoglicate. 
instantaneous breakup of the tear film simultaneously with the opening of the eye (FBUT and/or NIBUT; $0 \mathrm{~s}$ ). Although some patients showed a normal Schirmer test value ( $\geq 10 \mathrm{~mm} / 5 \mathrm{~min})$, their fluorescein breakup time was abnormal ( $\leq 5 \mathrm{~s})$. In Patient 10 , the spot-like fluorescein breakup of the precorneal tear film, which we coined as 'spot break' and that is observed instantaneously after opening the eye complicating the severest short BUT-type dry eye, ${ }^{38}$ was observed before starting the treatment with diquafosol.

\section{Subjective symptoms}

Changes in each symptom after the start of the $3 \%$ diquafosol ophthalmic solution treatment are shown in Figure 1. For all symptoms, the VAS score, except that for discharge, was moderately decreased during the observation period, and dryness, pain, and eye fatigue were markedly improved. At some of the observation points, difficulty in opening the eye, foreign body sensation, and redness were ameliorated in comparison to at baseline. There was a tendency for increased discharge at 1 month; yet, no statistically significant differences were observed throughout the observation period.

\section{Ocular-surface markers}

The tear meniscus radius, which is positively correlated with the cumulative tear volume in the conjunctival sac, averaged $0.172 \pm 0.063 \mathrm{~mm}$ before treatment with diquafosol sodium. It was significantly increased at 1 month after the start of the treatment, and the effectiveness of the treatment maintained for 12 months (Figure 2a). The results of the NIBUT and FBUT indicating index markers of the precorneal tear-film stability are shown in Figure $2 b$ and $c$, respectively. The baseline of NIBUT averaged $1.1 \pm 1.6 \mathrm{~s}$. The mean NIBUT was prolonged throughout the treatment period, and the maximum mean value was $4.5 \pm 2.2 \mathrm{~s}$ at 9 months after initiating the treatment (Figure 2b). The mean FBUT was similarly extended, and there were significant increases at $3,6,9$, and 12 months post treatment (Figure 2c). The tearfilm stability tended to elongate according to the treatment period. Patient 10 showed a 0 -s FBUT with the
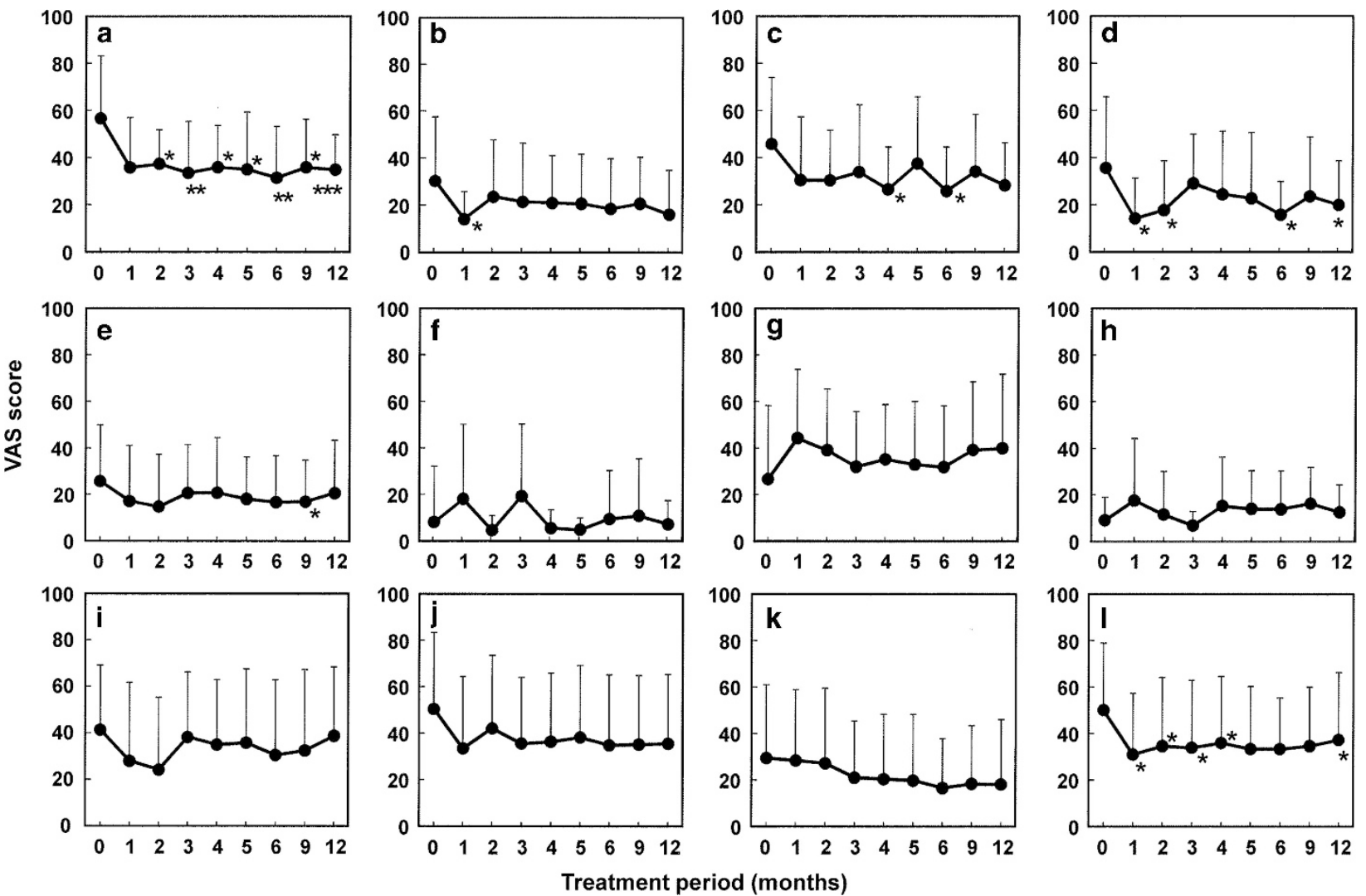

Figure 1 Change from baseline in each subjective symptom after the substitution of 3\% diquafosol ophthalmic solution for artificial tears and $0.1 \%$ sodium hyaluronate. Each graph shows the results of following symptoms; (a) dryness, (b) difficulty in opening the eye, (c) foreign body sensation, (d) pain, (e) redness, (f) tearing, (g) discharge, (h) itchiness, (i) blurred vision, (j) sensitivity to light, (k) heavy eyelids, (l) eye fatigue. Each data point represents the mean $\pm \mathrm{SD}$. ${ }^{*} P<0.05$, ${ }^{* *} P<0.01,{ }^{* * *} P<0.001$ vs baseline (0 months; Paired $t$-test). 


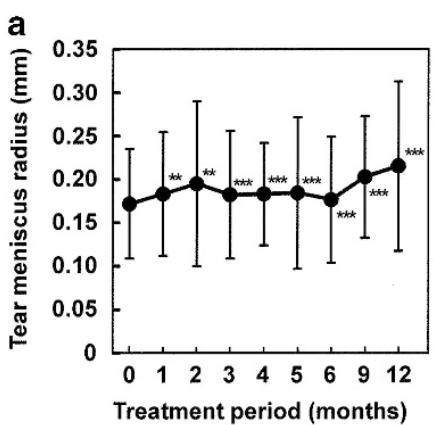

d

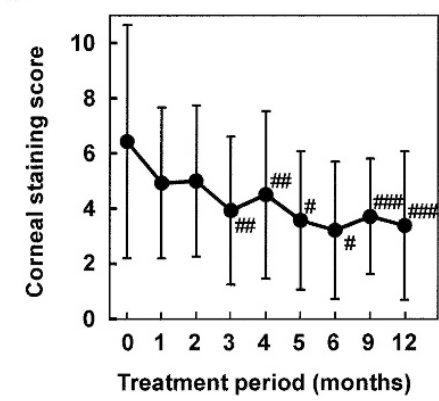

b

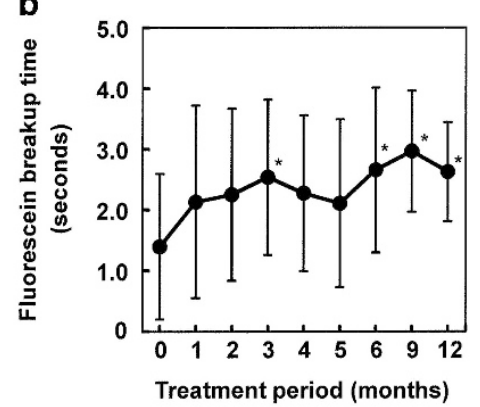

e

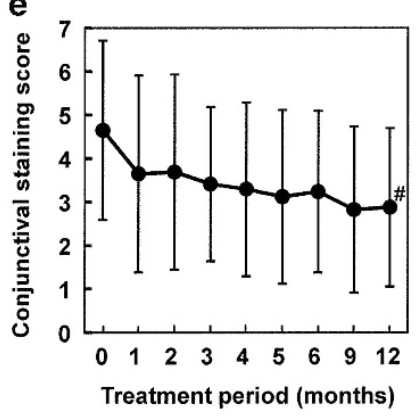

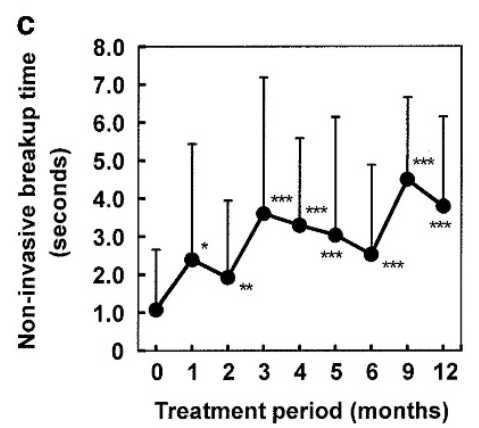

Treatment period (months)

Figure 2 Change from baseline in the ocular surface markers after the substitution of $3 \%$ diquafosol ophthalmic solution for artificial tears and $0.1 \%$ sodium hyaluronate. The tear meniscus radius (a) was increased, and tear-film instability ((b) noninvasive breakup time and (c) fluorescein breakup time) and the ocular-surface epithelial staining score ((d) corneal and (e) conjunctiva) was improved after the treatment with $3 \%$ diquafosol ophthalmic solution. Each data point represents the mean $\pm \mathrm{SD}$. ${ }^{*} P<0.05,{ }^{* *} P<0.01$, ${ }^{* * *} P<0.001$ vs baseline ( 0 months; Paired $t$-test). ${ }^{\#} P<0.05,{ }^{\#} P<0.01,{ }^{\# \#} P<0.001$ vs baseline ( 0 months; Wilcoxon's signed rank test).

spot-like tear-film breakup pattern at baseline, yet it was extended to 2 and $4 \mathrm{~s}$ at 5 and 12 months post treatment, respectively (Figure 3). Similarly, NIBUT was elongated from 0 to $10 \mathrm{~s}$. In addition, the ocular surface epithelial damage was found to have disappeared.

The results of the ocular-surface epithelial damage score are shown in Figure $2 \mathrm{~d}$ and e. The mean corneal epithelial damage score (as based on the National Institutes of Health guidelines) was $6.4 \pm 4.2$ at baseline and gradually decreased after the administration of diquafosol ophthalmic solution. Significant improvement was exhibited at 3 months, and its effectiveness continued throughout the observation period (Figure 2d). At baseline, the mean conjunctival epithelial staining score was $4.6 \pm 2.1$. That score improved, albeit very slowly, and significant improvement was observed at 12 months post treatment (Figure 2e). A representative case of aqueous tear-deficient dry eye is shown in Figure 4. The corneal and conjunctival epithelial staining scores were improved at 12 months post treatment. No adverse events were observed and none of the subjects discontinued their participation throughout the study period.

\section{Discussion}

The results of this study show that a 6-time daily treatment with $3 \%$ diquafosol ophthalmic solution in substitution for artificial tears and/or sodium hyaluronate effectively improves subjective symptoms and objective findings for a long-term period in dry-eye patients with SS. The needed frequency of the topical administration was less due to the relief of symptoms in comparison with that obtained from the existing therapy for dry eye with SS. In this study, the patients were administered artificial tears and sodium hyaluronate on the average of $9.8 \pm 3.0$ times (range: 7 to 16 times) per day prior to starting the 6-time daily application of diquafosol ophthalmic solution. Thus, the administration of diquafosol substitution proved to be advantageous from the aspect of reducing the burden on the patients.

All subjective symptoms were gradually alleviated after the start of the diquafosol treatment, and the VAS scores of major symptoms such as dryness, eye fatigue, and pain, were significantly decreased at from 1 to 2 months later. These results are in agreement with the findings of previous reports, ${ }^{20,22}$ thus illustrating that on average, the improvement of symptoms starts occurring during the early stage of the diquafosol treatment.

The subtle change of the tear volume in the conjunctival sac observed in the present study was detected throughout the diquafosol ophthalmic solution treatment period. Our findings are in agreement with those of the previous report showing that the change of tear volume was able to be detected using OCT after the treatment of 

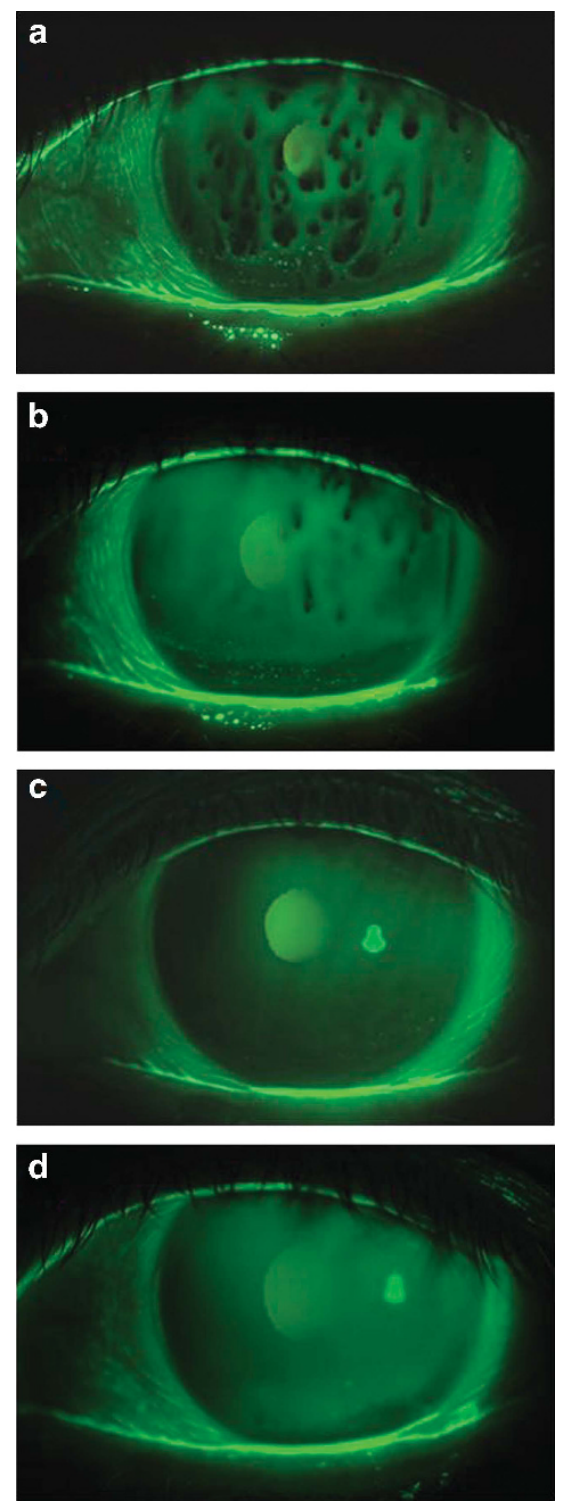

Figure 3 Slit-lamp photographs of the left eye of a 68-year-old female with short breakup time type dry eye with Sjögren's syndrome (SS). (a) The spot breakup pattern was simultaneously observed on the cornea when opening the eye before the start of the diquafosol administration (baseline). The spot breakup pattern had disappeared and the corneal epithelial staining was improved by the treatment with $3 \%$ diquafosol ophthalmic solution at 3, 6, and 12 months (b, c, and d, respectively) post treatment.

aqueous deficient dry eye with diquafosol. ${ }^{22}$ In the present study, we strictly controlled the timing of the tear meniscus radius measurements and performed those measurements at a greater than 1-h interval after the instillation of diquafosol ophthalmic solution. A single instillation of diquafosol reportedly can significantly increase tear volume for up to $30 \mathrm{~min}$, and thereafter, its effect returns to the baseline level. ${ }^{13}$ Therefore, the continuous tear volume increase may be attributed to the improvement of the water retention on the ocular surface epithelium by the diquafosol sodium induced membraneassociated mucin $^{16}$. Therefore, the possibility to increase the wettability and water retention ability on the ocular surface epithelium is theorized. However, further study is necessary to elucidate the exact mechanism of the increased baseline tear volume after the application of diquafosol.

BUT is regarded as a marker of tear-film instability, which is one of the core mechanisms of dry eye. ${ }^{1}$ In this study, both NIBUT and FBUT were gradually prolonged after the start of the diquafosol treatment, similar to the findings of the previous reports. ${ }^{18-22}$ These results suggest that mucin and tear fluid secretion induced by diquafosol improves the quality of tear film in dry-eye patients with SS. Diquafosol ophthalmic solution also improved corneal and conjunctival epithelial damage during the length of the treatment period. The corneal epithelial damage score was significantly decreased at 3 months after the initiation of the treatment, and that decrease was maintained.

However, the significant decrease of conjunctival damage was not observed until 12 months after the initiation of treatment. The reason why it took longer in this present study for the treatment to improve the conjunctival epithelial damage might be due to the fact that the study included many patients with severe dry eye. We previously reported that it was difficult to obtain improvement of conjunctival epithelial damage in SS by only increasing the fluid volume on the ocular surface. ${ }^{39}$ The severity of conjunctival damage in dry-eye patients with SS is greater than in those without SS, and numerous reports have described a variety of abnormalities such as increased inflammatory cytokine expression, decreased goblet cell density, and squamous cell metaplasia in the conjunctiva. ${ }^{4,6,8,40-42}$ Therefore, the effectiveness of diquafosol ophthalmic solution may be limited in the early stages of conjunctival improvement, as it has no anti-inflammatory capability. However, in the present study, from the point of ocular surface inflammation, any objective assessment was not performed for cellular or molecular biomarkers, which should potentially be examined in future studies.

In a previous report, $3 \%$ diquafosol ophthalmic solution exhibited similar efficacy to sodium hyaluronate in improving the fluorescein staining scores of dry-eye patients; yet, it exhibited superior efficacy in improving rose bengal staining scores. ${ }^{19}$ In this present study, substituting artificial tears and sodium hyaluronate with diquafosol ophthalmic solution produced similar results, thus suggesting that the 6-time daily application of diquafosol is superior to more frequent instillation of artificial tears and sodium hyaluronate. Assuming that the functions of lacrimal glands and mucin secretion from 

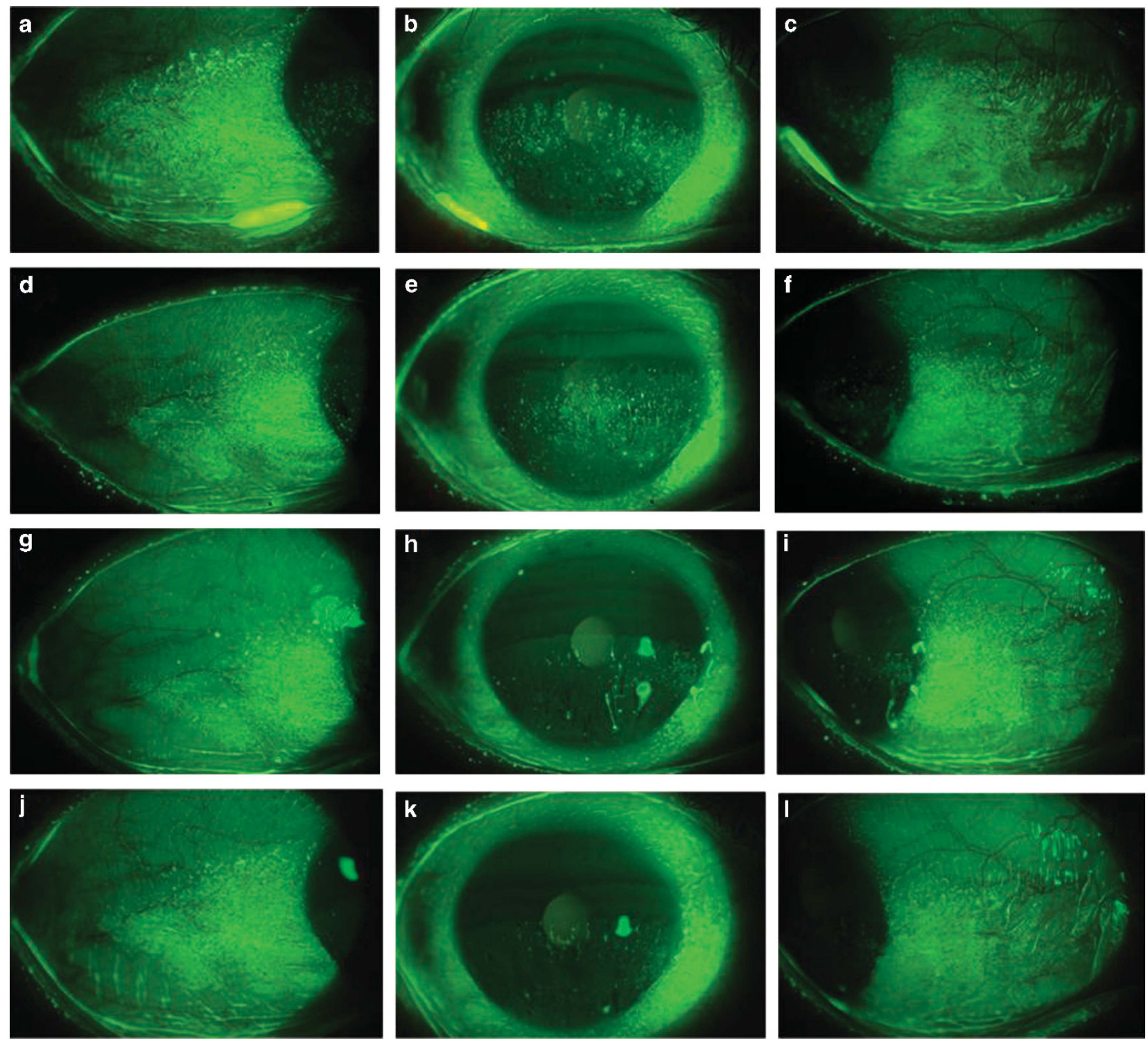

Figure 4 Slit-lamp photographs of the right eye of a 54-year-old female with aqueous tear-deficient dry eye with SS. The fluorescein staining of the cornea and conjunctiva was markedly improved at 6 and 12 months after the start of the treatment with $3 \%$ diquafosol ophthalmic solution in comparison to at baseline. At baseline: (a, b, and c); at 3 months: (d, e, and f); at 6 months: (g, h, and i); at 12 months: (j, k, and l). Nasal conjunctiva: (a, d, g, and j); cornea: (b, e, h, and k); temporal conjunctiva: (c, f, i, and l).

damaged conjunctival goblet cells was decreased, the improvement of signs and symptoms may have been the result of not only water secretion from conjunctival tissue but also the increased wettability of corneal epithelium via membrane-associated mucin expression. In addition, treatment by diquafosol ophthalmic solution has a distinct advantage over the treatment by artificial tears and sodium hyaluronate, as it provides longer water retention in the conjunctival sac. ${ }^{13,33}$

The majority of the enrolled subjects in this study were aqueous tear-deficient dry-eye patients; yet, 1 case of short BUT-type dry eye with severe symptoms was also included. However, this was a rare case, because dry eye with SS commonly is caused by lymphocyte infiltration and destruction of lacrimal glands, which leads to the decreased lacrimation. Previous reports suggested that short BUT-type dry eye is associated with allergic conjunctivitis $^{43}$ and a decreased mucin level in tear fluid. ${ }^{44}$ In fact, this case was complicated with perennial allergic conjunctivitis, so the manifestation of this case might have been caused by allergic conjunctivitis rather than lacrimal gland dysfunction. Diquafosol ophthalmic solution reportedly is effective in improving short BUTtype dry eye ${ }^{44}$ and this case also responded well to the 
diquafosol treatment. In addition to cases of short BUTtype dry eye, diquafosol ophthalmic solution reportedly was effective for improving the signs and symptoms in dry eye caused by a variety of risk factors such as laserassisted in situ keratomileusis, ${ }^{45,46}$ cataract surgery, ${ }^{47}$ and obstructive meibomian gland dysfunction. ${ }^{48}$ The multiple modes of action of diquafosol contribute to the improvement of tear-film stability, thus resulting in effectively treating dry eye.

In conclusion, for the treatment of dry eye with SS that received a topical application of artificial tears and sodium hyaluronate, substitution with 3\% diquafosol ophthalmic solution was found to be effective for 12 months for improving both subjective symptoms and objective findings. The findings of this study show that diquafosol ophthalmic solution is an effective basic therapeutic drug for the treatment of dry eye with SS.

\section{Summary}

What was known before

- Long-term results of 3\% diquafosol ophthalmic solution for the treatment of dry eye with Sjögren's syndrome is limitedly known.

\section{What this study adds}

- In dry-eye patients with Sjögren's syndrome, treatment with $3 \%$ diquafosol ophthalmic solution improved both symptoms and signs, and that effectiveness was maintained for 12 months.

\section{Conflict of interest}

NY is a consultant for Kissei Co., Ltd., Nagano, Japan and Rohto Co., Ltd, Osaka, Japan. SK is a consultant for Santen Pharmaceutical Co., Ltd, Osaka, Japan and Otsuka Pharmaceutical Co., Ltd., Tokyo, Japan. The remaining authors declare no conflict of interest.

\section{Acknowledgements}

We thank Mr John Bush (Senior Editor, Kyoto Prefectural University of Medicine) for reviewing the manuscript. This study was supported in part by Grants-in-Aid for scientific research from the Japanese Ministry of Education, Culture, Sports, Science and Technology.

\section{References}

1 The definition and classification of dry eye disease: report of the Definition and Classification Subcommittee of the International Dry Eye Work Shop. Ocul Surf 2007; 5: 75-92.

2 Fox RI. Sjögren's syndrome. Lancet 2005; 366: 321-331.
3 Tsubota K, Toda I, Yagi Y, Ogawa Y, Ono M, Yoshino K. Three different types of dry eye syndrome. Cornea 1994; 13: 202-209.

4 Kawasaki S, Kawamoto S, Yokoi N, Connon C, Minesaki Y, Kinoshita $\mathrm{S}$ et al. Up-regulated gene expression in the conjunctival epithelium of patients with Sjögren's syndrome. Exp Eye Res 2003; 77: 17-26.

5 Stern ME, Pflugfelder SC. Inflammation in dry eye. Ocul Surf 2004; 2: 124-130.

6 Hirai N, Kawasaki S, Tanioka H, Connon CJ, Yamasaki K, Yokoi $\mathrm{N}$ et al. Pathological keratinisation in the conjunctival epithelium of Sjögren's syndrome. Exp Eye Res 2006; 82: 371-378.

7 Caffery B, Simpson T, Wang S, Bailey D, McComb J, Rutka J et al. Rose bengal staining of the temporal conjunctiva differentiates Sjögren's syndrome from keratoconjunctivitis sicca. Invest Ophthalmol Vis Sci 2010; 51: 2381-2387.

8 Pflugfelder SC, Tseng SC, Yoshino K, Monory D, Felex C, Reis BL. Correlation of goblet cell density and mucosal epithelial membrane mucin expression with rose bengal staining in patients with ocular irritation. Ophthalmology 1997; 104: 223-235.

9 Argüeso P, Balaram M, Spurr-Michaud S, Keutmann HT, Dana RM, Gipson IK. Decreased levels of the goblet cell mucin MUC5AC in tears of patients with Sjögren's syndrome. Invest Ophthalmol Vis Sci 2002; 43: 1004-1011.

10 Fujihara T, Murakami T, Fujita H, Nakamura M, Nakata K. Improvement of corneal barrier function by the $\mathrm{P}_{2} \mathrm{Y}_{2}$ agonist INS365 in a rat dry eye model. Invest Ophthalmol Vis Sci 2001; 42: 96-100.

11 Yerxa BR, Douglass JG, Elena PP, Caillaud T, Amar T, Edick $\mathrm{C}$ et al. Potency and duration of action of synthetic $\mathrm{P}_{2} \mathrm{Y}_{2}$ receptor agonists on Schirmer scores in rabbits. Adv Exp Med Biol 2002; 506(Pt A): 261-265.

12 Murakami T, Fujita H, Fujihara T, Nakamura M, Nakata K. Novel noninvasive sensitive determination of tear volume changes in normal cats. Ophthalmic Res 2002; 34: 371-374.

13 Yokoi N, Kato H, Kinoshita S. Facilitation of tear fluid secretion by $3 \%$ diquafosol ophthalmic solution in normal human eyes. Am J Ophthalmol 2014; 157: 85-92.

14 Fujihara T, Murakami T, Nagano T, Nakamura M, Nakata K. INS365 suppresses loss of corneal epithelial integrity by secretion of mucin-like glycoprotein in a rabbit short-term dry eye model. J Ocul Pharmacol Ther 2002; 18: 363-370.

15 Takaoka-Shichijo Y, Sakamoto A, Nakamura M. Effect of diquafosol tetrasodium on MUC5AC secretion by rabbit conjunctival tissues. J Eye 2011; 28: 261-265.

16 Takaoka-Shichijyo Y, Nakamura M. Stimulatory effect of diquafosol tetrasodium on the expression of membranebinding mucin genes in cultured human corneal epithelial cells. J Eye 2011; 28: 425-429.

17 Nakamura M, Imanaka T, Sakamoto A. Diquafosol ophthalmic solution for dry eye treatment. Adv Ther 2012; 29: 579-589.

18 Matsumoto Y, Ohashi Y, Watanabe H, Tsubota KDiquafosol Ophthalmic Solution Phase 2 Study Group. Efficacy and safety of diquafosol ophthalmic solution in patients with dry eye syndrome: A Japanese phase 2 clinical trial. Ophthalmology 2012; 119: 1954-1960.

19 Takamura E, Tsubota K, Watanabe H, Ohashi Y Diquafosol Ophthalmic Solution Phase 3 Study Group. A randomised, double-masked comparison study of diquafosol versus sodium hyaluronate ophthalmic solutions in dry eye patients. Br J Ophthalmol 2012; 96: 1310-1315. 
20 Yamaguchi M, Tsubota K, Watanabe H, Ohashi Y. The safety and efficacy of long-term treatment with $3 \%$ diquafosol ophthalmic solution for dry eye. J Eye 2012; 29: 527-535.

21 Yamaguchi M, Nishijima T, Shimazaki J, Takamura E, Yokoi N, Wantanbe $\mathrm{H}$ et al. Clinical usefulness of diquafosol for real-world dry eye patients: a prospective, open label, non-interventional, observational study. Adv Ther 2014; 31: 1169-1181.

22 Koh S, Ikeda C, Takai Y, Watanabe H, Maeda N, Nishida K. Long-term results of treatment with diquafosol ophthalmic solution for aqueous-deficient dry eye. Jpn J Ophthalmol 2013; 57: 440-446.

23 Li Y, Kuang K, Yerxa B, Wen Q, Rosskothen H, Fischbarg J. Rabbit conjunctival epithelium transports fluid, and P2 $\mathrm{Y}_{2}$ receptor agonists stimulate $\mathrm{Cl}^{-}$and fluid secretion. $A m \mathrm{~J}$ Physiol Cell Physiol 2001; 281: C595-C602.

24 Murakami T, Fujihara T, Horibe Y, Nakamura M. Diquafosol elicits increases in net $\mathrm{Cl}^{-}$transport through $\mathrm{P}_{2} \mathrm{Y}_{2}$ receptor stimulation in rabbit conjunctiva. Ophthalmic Res 2004; 36: 89-93.

25 Shimmura S, Ono M, Shinozaki K, Toda I, Takamura E, Mashima Y et al. Sodium hyaluronate in the treatment of dry eyes. Br J Ophthalmol 1995; 79: 1007-1011.

26 Yokoi N, Komuro A, Nishida K, Kinoshita S. Effectiveness of hyaluronan on corneal epithelial barrier function in dry eye. Br J Ophthalmol 1997; 81: 533-536.

27 Fox RI, Robinson CA, Curd JG, Kozin F, Howell FV. Sjögren's syndrome. Proposed criteria for classification. Arthritis Rheum 1986; 29: 577-583.

28 Shimazaki J. Definition and diagnosis of dry eye 2006. J Eye 2007; 24: 181-184.

29 Toda I, Fujishima H, Tsubota K. Ocular fatigue is the major symptom of dry eye. Acta Ophthalmol (Copenh) 1993; 71: 347-352.

30 Yokoi N, Bron AJ, Tiffany JM, Kinoshita S. Reflective meniscometry: A new field of dry eye assessment. Cornea 2000; 19: S37-S43.

31 Oguz H, Yokoi N, Kinoshita S. The height and radius of the tear meniscus and methods for examining these parameters. Cornea 2000; 19: 497-500.

32 Yokoi N, Bron AJ, Tiffany JM, Maruyama K, Komuro A, Kinoshita S. Relationship between tear volume and tear meniscus curvature. Arch Ophthalmol 2004; 122: 1265-1269.

33 Yokoi N, Komuro A. A Non-invasive methods of assessing the tear film. Exp Eye Res 2004; 78: 399-407.

34 Ishibashi T, Yokoi N, Kinoshita S. Comparison of the shortterm effects on the human corneal surface of topical timolol maleate with and without benzalkonium chloride. I Glaucoma 2003; 12: 486-490.
35 Koh S, Watanabe H, Hosohata J, Hori Y, Hibino S, Nishida K et al. Diagnosing dry eye using a blue-free barrier filter. Am J Ophthalmol 2003; 136: 513-519.

36 Lemp MA. Report of National Eye Institute/Industry Work shop on clinical trials in dry eyes. CLAO J 1995; 21: 221-232.

37 van Bijsterveld OP. Diagnostic tests in the sicca syndrome. Arch Ophthalmol 1969; 82: 10-14.

38 Yokoi N, Georgiev GA. Tear-film-oriented diagnosis and therapy for dry eye. Yokoi N. Dry Eye Syndrome: Basic and Clinical Perspectives. Future Medicine: London, 2013; 96-108.

39 Hirotani Y, Yokoi N, Tsuzuki H, Kinoshita S. [Comparison of corneal and conjunctival rose bengal staining before and after punctal occlusion]. Nihon Ganka Gakkai Zasshi 2003; 107: 719-723.

40 Takamura E, Takano H, Yoshino K, Negoro K, Tsubota K, Kobayashi T. Quantitative cellular evaluation of conjunctival squamous metaplasia in the dry eye patient. Adv Exp Med Biol 1994; 350: 535-538.

41 Jones DT, Monroy D, Ji Z, Pflugfelder SC. Alterations of ocular surface gene expression in Sjögren's syndrome. Adv Exp Med Biol 1998; 438: 533-536.

42 Pflugfelder SC, Huang AJW, Feuer W, Chuchovski PT, Pereira IC, Tseng SCG. Conjunctival cytologic features of primary Sjögren's syndrome. Ophthalmology 1990; 97: 985-991.

43 Toda I, Shimazaki J, Tsubota K. Dry eye with only decreased tear break-up time is sometimes associated with allergic conjunctivitis. Ophthalmology 1995; 102: 302-309.

44 Shimazaki-Den S, Iseda H, Dogru M, Shimazaki J. Effects of diquafosol sodium eye drops on tear film stability in short BUT type of dry eye. Cornea 2013; 32: 1120-1125.

45 Masuda Q, Mori Y, Nejima R, Maruyama Y, Minami K, Miyata K. Efficacy of long-term treatment with diquafosol sodium for dry eye due to laser in situ keratomileusis. J Eye 2013; 30: 249-253.

46 Toda I, Ide T, Fukumoto T, Ichihashi Y, Tsubota K. Combination Therapy with diquafosol tetrasodium and sodium hyaluronate in patients with dry eye after laser in situ keratomileusis. Am J Ophthalmol 2014; 157: 616-622.

47 Takeshita T. Effect of diquafosol sodium ophthalmic solution for dry eye after cataract surgery. Jpn J Clin Ophthalmol 2013; 67: 327-330.

48 Arita R, Suehiro J, Haraguchi T, Maeda S, Maeda K, Tokoro $\mathrm{H}$ et al. Topical diquafosol for patients with obstructive meibomian gland dysfunction. $\mathrm{Br} J$ Ophthalmol 2013; 97: 725-729. 